FORMATION Formation emploi

Revue française de sciences sociales

122 | Avril-Juin 2013

Relever les défis de la Validation des Acquis de l'Expérience (VAE)

\title{
Edito : Valider ses acquis, une pratique à accompagner
}

Jean-Frédéric Vergnies

\section{OpenEdition}

Journals

Édition électronique

URL : http://journals.openedition.org/formationemploi/3978

DOI : 10.4000/formationemploi.3978

ISSN : 2107-0946

Éditeur

La Documentation française

Édition imprimée

Date de publication : 30 juin 2013

ISSN : 0759-6340

Référence électronique

Jean-Frédéric Vergnies, «Edito : Valider ses acquis, une pratique à accompagner », Formation emploi

[En ligne], 122 | Avril-Juin 2013, mis en ligne le 10 juillet 2013, consulté le 30 octobre 2020. URL

http://journals.openedition.org/formationemploi/3978 ; DOI : https://doi.org/10.4000/

formationemploi.3978

(c) Tous droits réservés 


\title{
Valider ses acquis, une pratique à accompagner
}

\author{
Jean-Frédéric Vergnies \\ Rédacteur en chef
}

T e dispositif de validation des acquis de l'expérience (VAE) vise à transformer l'expéLrience en équivalent formation afin d'obtenir un diplôme. Cette transformation est loin d'aller de soi ; s'engager dans la VAE est un processus qui se construit chemin faisant et nécessite un accompagnement.

Pour les candidats, selon P. Mayen \& J.-P. Pin "l'engagement en VAE, les processus par lesquels il se construit et fluctue, se renforce ou se délite, dépendent de la combinaison de nombreux facteurs non seulement propres à la VAE et à ses situations mais aussi aux personnes». Ils nous proposent d'examiner cette dynamique de l'engagement à la lumière de la didactique professionnelle, tandis que P. Saielli et M.-O. Legrand sollicitent la psychanalyse avec les travaux de Winnicott sur les objets transitionnels. Ainsi, pour certains candidats, il est difficile d'accéder à la dimension processuelle de la VAE, tandis que d'autres font preuve de créativité pour concilier les demandes de contenus et de processus, leur parcours en VAE est alors plus complexe mais aussi plus riche. Élaborer son expérience, aussi complexe que cela soit, ne suffit pas ; E. Auras nous explique qu'il faut aussi la mettre en scène, ce qui comporte d'autres enjeux, notamment pour les jurys.

Pour les accompagnateurs au coeur du dispositif et les jurys, il s'agit ensuite d'évaluer cette expérience et de décider de sa concordance avec la formation. Cela nécessite des ajustements et des arrangements au sein des jurys, comme le souligne S. Bellini. Cela dépend aussi du contexte. Au niveau régional, selon P. Rozario, la gestion stratégique de la VAE oriente la sélection initiale des candidats, et pèse sur les résultats au travers des consignes transmises aux membres de jury. On peut ainsi identifier des cultures différentes de la VAE. L'exemple du Portugal est également instructif. Ainsi, selon J. Caramelo et M. Santos, au travers de l'analyse du travail des formateurs-accompagnateurs, les enjeux apparaissent contradictoires ; d'une part, respecter les objectifs politiques d'une certification de masse et, d'autre part, construire une procédure en prenant en compte les par- 
ticularités du candidat et de son parcours. Enfin, dans la postface, Y. Clot et B. Prot reviennent sur les rapports entre référentiels et acquis à valider, et sur le rôle des collectifs de travail. Ils nous incitent à réinterroger la sécurisation des parcours professionnels, où la VAE pourrait jouer un rôle important, en remettant au cœur du débat l'activité des candidats, et leur travail sur leur métier.

Vincent Merle, ce nom aurait aussi pu figurer dans la liste des contributeurs à ce numéro. En effet, sans lui, nous ne l'aurions pas sous les yeux, et quelques mots ne suffiront pas à témoigner combien ce numéro lui doit, et à lui rendre hommage. Au secrétariat d'État à la Formation professionnelle, il a encouragé de manière décisive l'instauration de la VAE introduite par la loi en 2002. Accompagnateur et soutien indéfectible de Formation Emploi au travers de sa direction du Céreq et dans ses nombreuses autres fonctions avant et depuis, son parcours démontre, outre ses nombreuses autres qualités, combien le soutien et l'accompagnement sont d'autant plus précieux qu'ils sont prodigués dans la courtoisie et l'intelligence. Il aurait sans doute apprécié de lire, au travers de ces analyses, le déploiement tranquille de ce dispositif qu'il a contribué à construire et surtout ses effets collatéraux sur les représentations des métiers et des formations. Toute notre reconnaissance lui est acquise. 